\title{
Análisis multicriterio SIG basado en momentos de orden superior normalizados para el cálculo de superficies de viabilidad ambiental
}

\section{Multicriterion GIS analysis based on standardized higher-order times for calculating environmental viability surfaces}

\author{
Oscar Alberto Gómez Colorado \\ HYG CONSULTORES SAS \\ oscar.gomez1@gmail.com
}

\author{
Daniel Horfan Álvarez \\ HYG CONSULTORES SAS \\ dhorfan@gmail.com
}

\author{
Libardo Antonio Londoño Ciro \\ Universidad de San Buenaventura \\ libaranto@gmail.com
}

(Tipo de Artículo: Investigación Científica y Tecnológica. Recibido: 05/11/2017. Aprobado: 22/01/2018)

\begin{abstract}
Resumen. El presente estudio plantea una metodología para generar áreas de criticidad y niveles de susceptibilidad ambiental aptas para el trazado o localización de proyectos de infraestructura, con un alto grado de certidumbre comparado con los métodos convencionales basados en las funciones de máximos, media aritmética y promedio ponderado. La metodología se basa en la superposición espacial de variables físicas, ambientales y socioeconómicas que miden el impacto en el territorio ante el paso o ubicación del proyecto de infraestructura; aplicando el método de análisis estadístico denominado "momentos de orden superior" con un factor exponencial de normalización, propuesto por los autores. El modelo de análisis propuesto aplica principalmente en el Diagnóstico Ambiental de Alternativas (DAA) de proyectos lineales y concentrados, exigido por el Ministerio de Ambiente y Desarrollo Sostenible (MINAMBIENTE), en Colombia. El modelado espacial de los datos y las operaciones de superposición de capas de información, se realizaron a través de la herramienta Model Builder y la calculadora de mapas del software Arcgis. El análisis numérico y espacial realizado a través del SIG representa la interacción de los diferentes componentes bióticos, físicos y sociales (económicos, políticos y culturales), que inciden en la viabilidad del trazado o ubicación de un proyecto de infraestructura (vías, oleoductos, líneas de transmisión de energía, centrales hidroeléctricas, rellenos sanitarios, entre otros). Los resultados obtenidos demuestran como la superficie de susceptibilidad general, calculada con el método de momentos de orden superior normalizado, permite una toma de decisiones adecuada, toda vez que puede ajustarse con mayor aproximación a las diferentes restricciones ambientales de la región.
\end{abstract}

Abstract. This study presents a methodology to generate critical areas and levels of environmental susceptibility for design projects of linear infrastructure, or the location of concentrated projects, with a high degree of certainty compared to conventional methods based on the functions of maximum, arithmetic and weighted averages. The methodology is based on the calculation of surfaces susceptibility and combination of environmental variables in the territory, applying the statistical probability method called "higher order moments" with an exponential normalization factor proposed by the authors; combined with spatial analysis tools provided by Geographic Information Systems (GIS). The proposed method applies mainly in the Environmental Assessment of Alternatives (DAA) of linear and focused projects, required by the Ministry of Environment and Territorial Development (Minambiente) in Colombia. Spatial modeling of data and operations overlapping layers of information, were carried through the Model Builder tool and map calculator of the software Arcgis. The numerical and spatial analysis through GIS represents the interaction between the different biotic, physical and social components that affect the viability of project infrastructure (roads, pipelines, lines power transmission, hydropower plants, landfills, etc.).

The results show the surface as general susceptibility calculated with method of normalized higher order moments, allows appropriate decision-making, since most closely can be adjusted to different environmental restrictions of the region.

Palabras clave. DAA, Análisis Multicriterio, Momentos de Orden Superior, SIG, Análisis Espacial, Modelo Builder.

Keywords. DAA, Multicriterial Analysis, Higher Order Moments, GIS Spatial Analysis, Model Builder.

DOI 10.21500/20275846.3300 


\section{INTRODUCCIÓN}

El Diseño de proyectos de infraestructura lineales o concentrados requiere de estudios técnicos y ambientales que permitan establecer las áreas de mayor viabilidad para el trazado de la ruta. Los Sistemas de Información Geográfica (SIG) son una importante herramienta para la planificación ambiental y ordenamiento del territorio [2], al permitir generar superficies de susceptibilidad mediante la superposición de capas temáticas en formato raster (matriz de celdas), las cuales recogen los criterios de evaluación ambiental necesarios en la valoración de impactos sobre el área del proyecto [3]. La superposición de mapas permite interrelacionar las variables técnicas del diseño con las variables ambientales, con el fin de producir criterios de decisión que conduzcan a la obtención de las mejores alternativas de trazado para proyectos lineales $[4,6]$.

El cálculo de niveles de susceptibilidad mediante la superposición de mapas, se realiza comúnmente a partir de funciones estadísticas que retornan el valor máximo ó el promedio aritmético o ponderado de las celdas. Estos métodos presentan un alto nivel de incertidumbre, por cuanto no le dan la verdadera importancia a las variables que pueden tener un peso importante en la decisión; o en otros casos, no tienen en cuenta el peso de todas las variables para el cálculo de la superficie de susceptibilidad final.

Buscando resolver esta incertidumbre, se realizó un análisis sobre un proyecto hipotético en el Norte y Nordeste de Antioquia (Colombia), para estudiar la respuesta de otros métodos matemáticos que permitieran obtener superficies de susceptibilidad más cercanas a la realidad del terreno. Entre los métodos evaluados, se encontró que la función estadística de Distribución de Momentos de Orden Superior, aplicándole un factor de potencia $(\sqrt[k]{ })$ propuesto por los autores, calcula el promedio de los datos de susceptibilidad, dándole mayor sensibilidad e importancia a las variables más críticas. A este método se le ha denominado Momentos de Orden Superior Normalizado.

El factor de potencia adicionado a la ecuación de momentos de orden superior, puede ser ajustado de acuerdo a las condiciones ambientales de la región (ver numeral 2.6).

Este documento, contempla los aspectos conceptuales, formulación y el análisis de resultados con el método propuesto.

\section{Marco conceptual}

\subsection{Modelo de susceptibilidad Ambiental}

La capacidad de asimilación y respuesta de los sistemas ambientales ante la implementación de un proyecto, indican la condición o estado de sensibilidad de estos frente a los impactos generados. El análisis de susceptibilidad ambiental mide el grado de vulnerabilidad que presentan estos sistemas y que reflejan de alguna forma, la muy baja o ninguna capacidad de asimilación [4, 14].

El modelo de susceptibilidad ambiental considera también todos aquellos factores que, por sus características particulares, determinan la no viabilidad de un proyecto, sobre un área geográfica específica. En este grupo se encuentran las áreas de reserva forestal, parques nacionales naturales, cuerpos de agua, centros poblados, entre otros. Los factores que determinan la susceptibilidad ambiental pueden determinarse a partir de:

- La caracterización de los factores ambientales

- Los distintos grados de vulnerabilidad observables

- La complejidad y costos de la gestión ambiental

Según los grados de vulnerabilidad habrá diferentes rangos y niveles de susceptibilidad ambiental así [1]:

- Susceptibilidad muy baja: el proyecto no ocasiona ningún efecto, modificación o impacto al entorno.

- Susceptibilidad baja: los posibles impactos pueden manejarse mediante la implementación de medidas generales de manejo ambiental.

- Susceptibilidad media: los impactos generados son de orden reversible y su atención requiere la implementación de medidas de manejo ambiental de carácter específico.

- Susceptibilidad alta: Las medidas para atender los posibles efectos son de carácter local y proporcionalmente de mayores especificidades, generando mayores costos en la gestión ambiental.

- Susceptibilidad muy alta: zonas con gran sensibilidad $y$ efectos de gran deterioro sobre el ambiente que dificultan el paso de la línea de transmisión. Los efectos ocasionados requieren medidas de manejo ambiental locales especiales y muy detalladas.

- Restricción: zonas en las cuales no existe posibilidad alguna de implementación del proyecto, debido a la irreversibilidad de los impactos y a la legislación ambiental que las considera en la mayoría de los casos, áreas de reserva.

\subsection{Análisis por Componentes Analíticas}

El análisis de susceptibilidad ambiental involucra el concepto de componente analítica para agrupar todos aquellos factores con particularidades propias dentro de un entorno común. El modelo de susceptibilidad ambiental debe considerar por norma tres componentes analíticas [1]:

- Abiótica: Agrupa los criterios definidos por las condiciones del terreno y sus geoformas.

- Biótica: Agrupa los criterios definidos por las condiciones de la flora, fauna y ecosistemas estratégico.

- Cultural: Agrupa los criterios definidos por las condiciones socioculturales y económicos de la región. 


\subsection{Asignación del rango de susceptibilidad}

Con la participación de un equipo interdisciplinario de profesionales asociados a las distintas dimensiones, se establece una escala estandarizada de valores que definen los criterios de susceptibilidad y restricción ambiental de cada una de las variables.

El rango de variación de los valores de susceptibilidad se estableció entre 1 y 6 , siendo 1 el valor de menor susceptibilidad y 5 el valor de máxima susceptibilidad, los valores de 6 o No Data, corresponden a las áreas restrictivas por donde, en ningún caso, el proyecto podrá cruzar.

La escala de valoración tiene como objetivo dar una representación numérica a los diferentes criterios de evaluación usados en el análisis ambiental, con el fin de poder llevarlos al Sistema de Información Geográfica y mediante una operación de superposición de capas, aplicarles la formulación matemática propuesta para la susceptibilidad ambiental, tanto para cada componente como para el conjunto de ellas. La Tabla 1 muestra la descripción para cada rango de susceptibilidad empleado.

Tabla 1. Valores asignados por susceptibilidad ambiental

\begin{tabular}{ll}
\multicolumn{2}{c}{ ambibilidad } \\
\hline 1 & Susceptib \\
2 & Muja baja \\
3 & Media \\
4 & Alta \\
5 & Muy alta \\
6 & Restrictiva \\
\hline
\end{tabular}

\subsection{Cálculo de Susceptibilidad Ambiental}

Una vez se tiene la calificación de las variables dentro del rango de susceptibilidad establecido, se calculan los raster o superficies ${ }^{1}$ de susceptibilidad para cada una de las componentes ambientales evaluadas. Este proceso debe repetirse para el cálculo de la susceptibilidad general, empleando como variables de entrada las superficies de susceptibilidad por componente, la cual finalmente servirá de insumo para el cálculo de alternativas.

El cálculo de superficies de susceptibilidad se realiza normalmente mediante la superposición de mapas, a partir de funciones estadísticas que retornan el valor máximo, el promedio aritmético o el promedio ponderado de las celdas.

\subsubsection{Método de Promedio Aritmético}

También conocido como media aritmética, retorna el valor medio de un conjunto de datos cuantitativos a partir de la suma de todos sus valores divido entre el total de datos. Su formulación matemática para el cálculo de

\footnotetext{
1 Superficie: Término asignado en Sistemas de Información Geográfica a los datos geográficos conformados por matrices de celdas (datos raster).
}

susceptibilidades viene definida por la siguiente ecuación 1:

$$
S_{m}=\frac{1}{n} \sum_{i=1}^{n}\left(X_{i}\right)
$$

Donde:

$$
\begin{aligned}
& S_{m}: \text { Susceptibilidad media por componente } \\
& X_{i}: \text { Variable analítica } \\
& n: \text { Número de variables }
\end{aligned}
$$

El método de promedio aritmético entrega una superficie cuya mayor parte del área contiene valores de susceptibilidad medios a bajos, debido a que los valores altos de criticidad pueden ser atenuados por las celdas de menor valor.

\subsubsection{Método de Promedio Ponderado}

Se superponen los mapas raster de cada componente analítica usando una escala de susceptibilidad común y un peso, de acuerdo a su importancia. Para el cálculo de la susceptibilidad por componente, la ecuación viene dada por:

$$
S p=\frac{1}{n} \sum_{i=1}^{n}\left(X_{i} . P i\right)
$$

Donde:

$$
\begin{aligned}
& S_{p}: \text { Susceptibilidad ponderada } \\
& X_{i}: \text { Variable analítica } \\
& P_{i}: \text { Peso de la variable } \\
& n: \text { Número de variables }
\end{aligned}
$$

El peso dado a cada variable de análisis depende de su importancia en la decisión. Su valor va de 1 al $100 \%$.

\subsubsection{Método de Máximos}

El método de máximos evalúa los valores de susceptibilidad entre celdas espacialmente coincidentes de cada raster temático y escoge la de mayor valor. La formulación matemática del modelo de máximos para el cálculo de la susceptibilidad por componente viene dado por la ecuación 3:

$$
\operatorname{Smax}=\operatorname{Max}\left(x_{i}, x_{j}, x_{k}, \ldots, x_{n}\right)
$$

Donde:

$$
\begin{aligned}
& S_{\text {max }}: \text { Susceptibilidad por máximos } \\
& x_{i, j, k, . . n: \text { Variable analítica }} \\
& \text { Max: Función de Máximos. }
\end{aligned}
$$

El principal inconveniente de este método es que sólo tiene en cuenta la variable de mayor valor, sin tener en cuenta la importancia que puedan tener las demás 
variables en la decisión. Como consecuencia, entrega superficies de viabilidad bastante costosas que muestran muy crítico el desarrollo del proyecto. Adicionalmente, no refleja la diferencia entre dos zonas de alta susceptibilidad.

\subsubsection{Momentos de Orden Superior (MOS)}

Los momentos de orden superior son funciones estadísticas de distribución de probabilidad que pueden estar centradas en el origen o en la media.

Cuando se tiene una distribución discreta, los momentos de orden superior centrados en el origen, vienen dados por la ecuación 4 [14]:

$$
m_{k}=\sum_{i=1}^{n} x_{i}^{k} \cdot p\left(x_{i}\right)
$$

Donde:

$$
\begin{aligned}
& m_{k}: \text { Momento de Orden Superior } K \\
& k: \text { Numero de Orden } \\
& \text { Xi: Conjunto de variable } \\
& p(x i): \text { Probabilidad de Xi } \\
& n: \text { Numero de variables }
\end{aligned}
$$

Cuando están centrados en la media, están dados por:

$$
m_{k}=\sum_{i=1}^{n}\left(x_{i}-\bar{x}\right)^{k} \cdot p\left(x_{i}\right)
$$

Donde:

$$
\begin{gathered}
\overline{\boldsymbol{x}} \text { : Media del Conjunto de valores, dada por: } \\
\sum_{x_{i} / n}
\end{gathered}
$$

Los momentos de orden superior tienen varias aplicaciones en estadística, por ejemplo: el momento de orden 2 centrado en la media permite calcular la desviación estándar y la varianza [18], mientras que en física es la base para el cálculo del momento de inercia [20].

El momento de orden 3 centrado en la media, también es llamado función de Skewness y se utiliza para calcular el coeficiente de asimetría de un conjunto de datos que tienen una distribución normal [19].

El momento de orden 4 centrado en la media, se usa para el cálculo del coeficiente de Kurtosis, que permite calcular el grado de aplanamiento de una distribución normal [19] (ver Figura 1).

$$
\sigma=\sqrt{\frac{\sum_{i=1}^{N}\left(x_{i}-\bar{x}\right)^{2}}{N}}
$$

Desviación Estándar

$$
A=\frac{m_{3}}{\sigma^{3}}
$$

Coeficiente de momento de Asimetría

$$
\sigma^{2}=\frac{\sum_{i=1}^{N}\left(x_{i}-\bar{x}\right)^{2}}{N}
$$

Varianza

$$
\mathrm{K}=\frac{m_{4}}{\sigma^{4}}
$$

Coeficiente de Kurtosis

Figura 1. Ecuaciones para el uso de momentos de Orden Superior en Estadística

\subsubsection{Momentos de Orden Superior Normalizados}

El método MOS eleva la importancia de la variable $X$ proporcionalmente a su peso, no obstante, cuando se emplea una escala de valoración específica (tal como la escala de susceptibilidad mencionada anteriormente, Tabla 1), los valores obtenidos no son fácilmente interpretables. Por lo anterior, fue necesario incorporar un factor exponencial de normalización en la ecuación (4), para garantizar que los resultados a ajustaran a este rango. Este factor viene dado por la siguiente expresión:

$$
S=\sqrt[k]{\sum_{i=1}^{n} x_{i}^{k} \cdot p\left(x_{i}\right)}
$$

donde:

$$
\begin{aligned}
& \text { k: Factor de potencia o normalización } \\
& \text { xi: Variable analítica } \\
& \text { p: Peso dado a la variable analitica } \\
& \text { n: Número de variables } \\
& \text { S: Susceptibilidad }
\end{aligned}
$$

El factor de potencia $(\sqrt[k]{ })$ adicionado a la ecuación de momentos de orden superior (4), puede ser ajustado de acuerdo a las condiciones ambientales de la región. De esta forma, en condiciones adversas, el valor de potencia asume valores altos para darle importancia a las variables más críticas, y en condiciones ambientalmente favorables, este factor puede tomar valores bajos. Lo anterior, conservando la escala de valoración empleada en el análisis.

\section{Cálculo susceptibilidades usando momentos de orden superior normalizados}

En el modelo propuesto, veremos como la variación del exponente $\mathrm{k}$, puede enfatizar la importancia de algunas variables o contrarrestar el efecto de otras, de acuerdo a su peso en el análisis realizado.

Partiendo de la ecuación 7 se obtiene la siguiente expresión para el modelo de susceptibilidad general 


$$
S g=\sqrt[k]{\sum_{i=1}^{n} T_{i}^{k} \cdot p_{i}}
$$

Donde:

Sg: Susceptibilidad General

$k$ : Numero de Orden

Ti: Susceptibilidad de la variable temática o de la componente ambiental

Pi: Peso o importancia dado a la variable

$N$ : Numero de variables

En esta ecuación, un valor alto de k proporciona una mayor relevancia a los valores de susceptibilidad de las variables críticas y, lo contrario, un valor de $k$ pequeño contrarresta el efecto de las variables críticas durante el análisis.

Cuando todas las variables tienen el mismo peso o importancia en la decisión, la función toma la siguiente forma:

$$
S g=\sqrt[k]{\frac{\sum_{i=1}^{n} T_{i}^{k}}{n}}
$$

\subsection{Selección del Numero de Orden k}

En el modelo propuesto, la selección adecuada de un valor de $\mathrm{k}$ es vital y depende de la decisión del experto ambiental. La tabla 2 contiene los resultados comparativos de susceptibilidad general entre los métodos de Máximos, Promedios Ponderados y el modelo propuesto, para diferentes valores de k. La superposición se hace con tres celdas de igual peso, manteniendo constante, en las dos primeras, la susceptibilidad con los valores extremos de la escala (5 y 1 , respectivamente) e incremental de 1 a 5 , en la última.

Tabla 2. Comparación de Resultados para diferentes valores de $\mathrm{K}$

\begin{tabular}{llllllllll}
\hline $\begin{array}{l}\text { Valores } \\
\text { Celda }\end{array}$ & de & \multicolumn{1}{l}{ Resultados con diferentes Modelos } \\
\hline $\mathrm{T}$ & $\mathrm{T}$ & $\mathrm{T}$ & Pro & Máx & $\mathrm{K}: 2$ & $\mathrm{~K}: 3$ & $\mathrm{~K}: 4$ & $\mathrm{~K}: 5$ & $\mathrm{~K}: 1$ \\
1 & 2 & 3 & $\mathrm{~m}$ & & & & & 0 \\
5 & 1 & 1 & 2.3 & 5.0 & 3.0 & 3.5 & 3.8 & 4.0 & 4.5 \\
5 & 1 & 2 & 2.7 & 5.0 & 3.2 & 3.5 & 3.8 & 4.0 & 4.5 \\
5 & 1 & 3 & 3.0 & 5.0 & 3.4 & 3.7 & 3.9 & 4.1 & 4.5 \\
5 & 1 & 4 & 3.3 & 5.0 & 3.7 & 4.0 & 4.1 & 4.2 & 4.5 \\
5 & 1 & 5 & 3.7 & 5.0 & 4.1 & 4.4 & 4.5 & 4.6 & 4.8 \\
\hline
\end{tabular}

En la Figura 2, se muestran los resultados de la tabla anterior en forma gráfica. Se observa que las curvas de

\footnotetext{
2 Cada uno de estos criterios debe ser medido a partir de la información cartográfica y temática disponible en el área de estudio, definida entre los dos puntos de conexión del proyecto. Para tal efecto se hace necesario realizar un inventario de información disponible a la escala exigida para este tipo de estudios (1:100.000), o menor en el mejor de los casos. Las posibles fuentes de información, de carácter
}

susceptibilidad del modelo propuesto se ubican entre las obtenidas por el método de máximos y el de promedios. Al aumentar el valor de $\mathrm{K}$, el método de momentos de orden superior, se acerca al resultado de máximos, sin perder la representatividad de los otros datos.

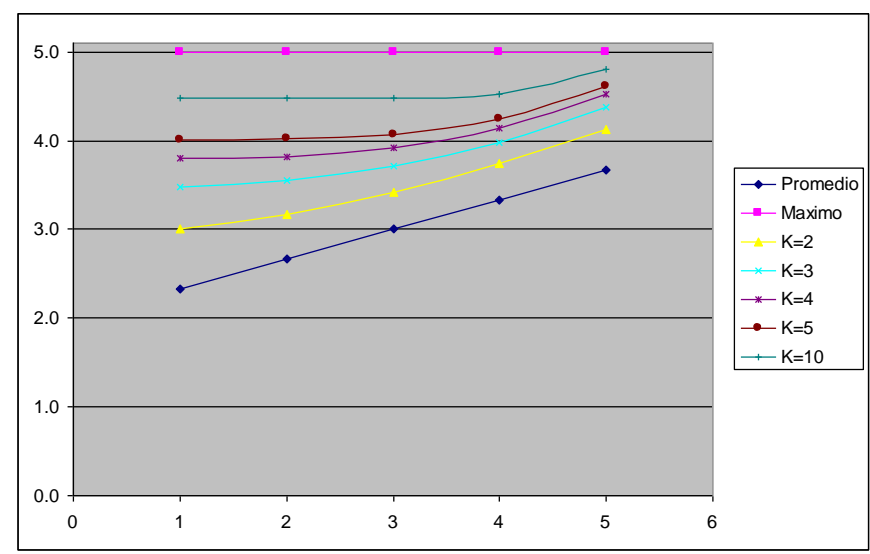

Figura 2. Comparación de Resultados diferentes valores de $\mathrm{K}$

\subsection{Proyecto Piloto}

La validación del modelo se realizó en un área piloto en la región del bajo cauca y norte de Antioquia, comprendida entre los municipios de Gómez Plata y Zaragoza. En este estudio se evaluó la influencia del valor del número de orden $\mathrm{K}$ sobre el cálculo del mapa de susceptibilidad general usado para definir los corredores de viabilidad ambiental con miras a la construcción de una carretera que comunique estos dos municipios. El componente ambiental se calculó basado en el modelo por dimensiones explicado arriba.

Con el fin de facilitar la interpretación visual de los resultados, se utilizó la gama de colores mostrada en la Figura 3:

\begin{tabular}{|l|l|}
\hline & Susceptibilidad Muy Baja \\
\hline & Susceptibilidad Baja \\
\hline & Susceptibilidad Media \\
\hline & Susceptibilidad Alta \\
\hline & Susceptibilidad Muy Alta \\
\hline & Áreas restrictivas \\
\hline
\end{tabular}

Figura 3. Simbología mapas de susceptibilidad

Los criterios empleados para la evaluación del proyecto se tomaron de los establecidos por la autoridad ambiental para este tipo de proyectos [1]. De igual forma, se identificaron las capas de información disponibles para cada criterio ${ }^{2}$, a escala 1:100.000. Las tablas 3, 4 y 5 describen la información correspondiente:

público, disponibles para Antioquia pueden ser adquiridas de entidades como el IGAC (Instituto Geográfico Agustín Codazzi, IDEAM (Instituto de Hidrología, Meteorología y Estudios Ambientales de Colombia), Corporaciones Ambientales (en este caso CORANTIOQUIA), Gobernación de Antioquia, entre otros. 


\subsubsection{Definición de Criterios}

\subsubsection{Componente Abiótico}

Agrupa los criterios definidos por las condiciones del terreno y sus geoformas.

Tabla 3. Criterios del Componente Abiótico

\begin{tabular}{lll}
\hline Criterio & & Capa de información \\
\hline $\begin{array}{l}\text { Zonas con pendiente excesiva, } \\
\text { propensas a erosión o a } \\
\text { inestabilidad }\end{array}$ & Raster de pendientes \\
\hline $\begin{array}{l}\text { Zonas de riesgo natural establecidas } \\
\text { a nivel nacional, regional y local }\end{array}$ & $\begin{array}{l}\text { Amenaza por Movimientos } \\
\text { de Masa e Inundación }\end{array}$ \\
\hline $\begin{array}{l}\text { Afectación mínima de áreas } \\
\text { pobladas } \\
\text { Afectación mínima de los cuerpos de } \\
\text { agua }\end{array}$ & $\begin{array}{l}\text { Densidad de Construcción } \\
\text { Zonas de inestabilidad Geológica de Agua }\end{array}$ \\
& $\begin{array}{l}\text { Fallas, Geomorfología } \\
\text { Geología, }\end{array}$ \\
\hline
\end{tabular}

\subsubsection{Componente Biótico}

Agrupa los criterios definidos por las condiciones de la flora, fauna y ecosistemas estratégicos. Las capas de información a evaluar en el ejercicio incluyen:

Tabla 4. Criterios del Componente Biótico

\begin{tabular}{lll}
\hline Criterio & & Capa de información \\
\hline $\begin{array}{l}\text { Áreas de exclusión o manejo } \\
\text { especial del orreas de Reserva, PNN } \\
\text { regional nacional o }\end{array}$ & \\
\hline $\begin{array}{l}\text { Áreas de alta importancia para la } \\
\text { preservación de la biodiversidad y/o } \\
\text { prioritarias para la conservación del } \\
\text { recurso faunístico }\end{array}$ & \\
\hline $\begin{array}{l}\text { Ecosistemas } \\
\text { legalmente definidos estratégicos }\end{array}$ & Área de interés ambiental \\
\hline $\begin{array}{l}\text { Áreas de reserva de la Ley } 2 \text { de } \\
\text { 1959, áreas de manglares, áreas } \\
\text { declaradas como zonas Ramsar. }\end{array}$ & Ley 2a \\
\hline
\end{tabular}

\subsubsection{Componente Cultural}

Agrupa los criterios definidos por las condiciones socioculturales y económicos de la región. Las capas de información a evaluar por cada criterio son las siguientes:

\section{Tabla 5. Criterios del Componente Cultural}

\begin{tabular}{lll}
\hline \multicolumn{1}{c}{ Criterio } & \multicolumn{1}{c}{ Capa de información } \\
\hline $\begin{array}{l}\text { Zonas donde el proyecto pueda } \\
\text { generar conflictos con el uso del }\end{array}$ & Conflictos del suelo \\
suelo. & & \\
\hline $\begin{array}{l}\text { Zonas en donde el recurso hídrico } \\
\text { sea escaso y el proyecto pueda }\end{array}$ & \\
ocasionar conflictos de uso. & \\
\hline $\begin{array}{l}\text { Zonas pobladas. } \\
\text { Sitios de reconocido interés }\end{array}$ & Patrimonio Arqueológico \\
histórico, cultural y arqueológico, \\
declarados como parques
\end{tabular}

\subsubsection{Calculo de superficies de viabilidad}

El cálculo de superficies de viabilidad se obtuvo integrando las variables temáticas por cada componente analítica a partir de los métodos estadísticos citados arriba (ecuaciones 1, 2, 3 y 7). Para tal efecto se requiere hacer una conversión de los datos geográficos de formato vectorial a raster, con el fin de realizar las operaciones de superposición celda a celda a través de la calculadora raster (raster calculator de Arcgis). Esta conversión se realiza a partir del campo de clasificación de susceptibilidad ambiental de acuerdo a la escala de valoración establecida en la tabla 1. El tamaño de las celdas depende de la escala de los datos fuente, por ejemplo, para escalas 1:25.000 (escala de diseño) se recomienda un tamaño de celda de 20 metros y para escala 1: 100.000 (escala de factibilidad) de 80 a $100 \mathrm{~m}$.

Posteriormente, debe llevarse a cabo un proceso de reclasificación para garantizar que las celdas que hayan sido calificadas con un valor restrictivo (6) sean convertidas a NoData, lo cual garantiza que, al realizar los cálculos de superposición, estas áreas no sean tenidas en cuenta en el trazado o ubicación del proyecto.

El modelo de cálculo se elaboró en el software de sistemas de información geográfica Arcgis, empleando la herramienta Model Builder con funciones de análisis raster y algebra de mapas.

Deberán calcularse inicialmente las superficies de viabilidad para cada componente ambiental: biótica, abiótica, cultural, socioeconómica, entre otras; y finalmente, la superficie de viabilidad general, combinando las superficies obtenidas para cada componente, empleando la calculadora raster y cualquiera de los métodos citados en los numerales 2.4.1 a 2.4.5.

\subsubsection{Resultados}

Las figuras 4, 5 y 6 muestran los resultados del mapa de susceptibilidad ambiental, obtenidos con los métodos convencionales de promedio aritmético, ponderado y máximos; respectivamente. Nótese los altos valores de criticidad (rojos) entregados por el método de máximos, mientras que el método de promedios aritmético arroja una superficie con valores de susceptibilidad intermedios (amarillos) y bajos (verdes) muy característico de este método debido a la atenuación que producen las celdas de menor valor sobre aquellas de valor medio y alto. La superficie obtenida con el método de promedio ponderado incrementa los valores de susceptibilidad en una cantidad importante de celdas; debido principalmente al mayor peso dado al componente biótico (para este ejemplo se emplearon los siguientes pesos: $50 \%$ para el componente Biótico, $30 \%$ para el Abiótico y $20 \%$ para el Cultural). 


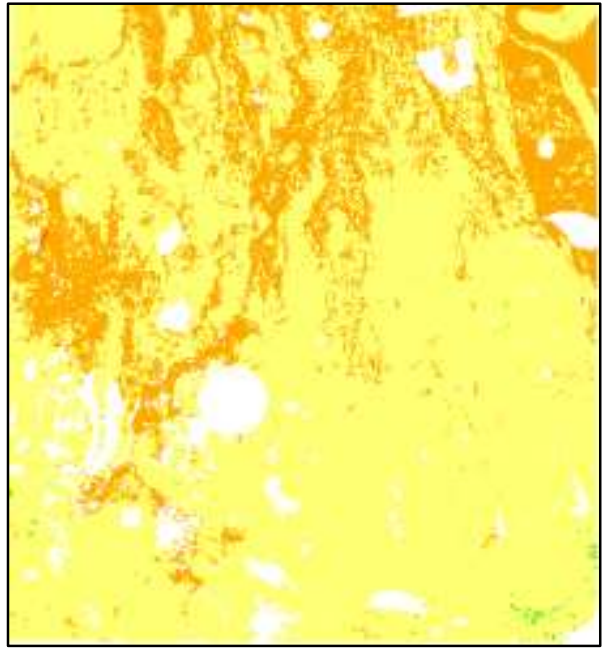

Figura 4. Susceptibilidad General con promedio aritmético.

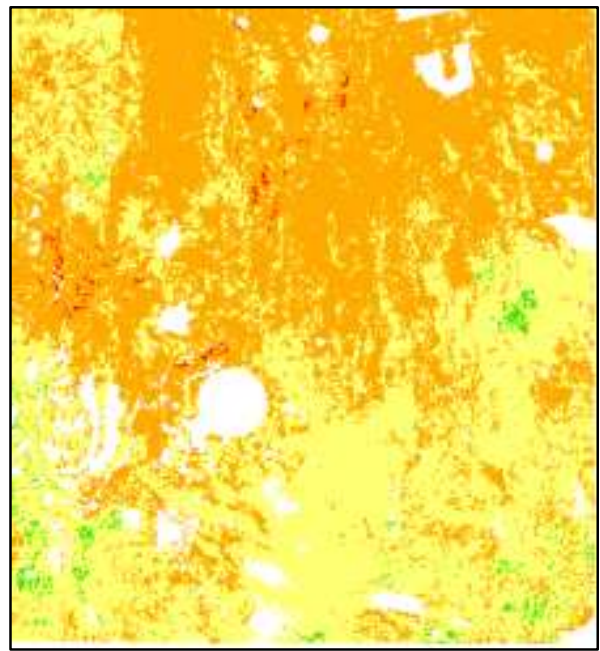

Figura 5. Susceptibilidad General con promedio ponderado.

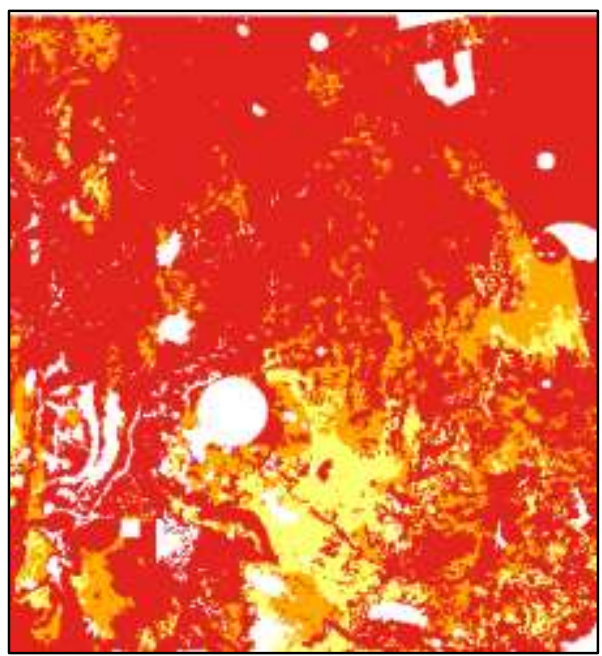

Figura 6. Susceptibilidad General con máximos.

\begin{tabular}{|l|l|}
\hline \multicolumn{2}{|c|}{ Susceptibilidad } \\
\hline & Muy Baja \\
\hline & Baja \\
\hline Media \\
\hline Alta \\
\hline Muy Alta \\
\hline
\end{tabular}

Figura 7. Cuadro de convenciones

La Figuras 8, 9 y 10 muestran los resultados de susceptibilidad ambiental para varios valores de $\mathrm{k}(2,5$ y 10) empleando el método de momentos de orden superior normalizado. Se observa que a medida que el valor de $\mathrm{k}$ aumenta, los valores de susceptibilidad se vuelven más críticos, tendiendo a la superficie de máximos, tal como se explicó en el numeral 3.1, Figura 2.

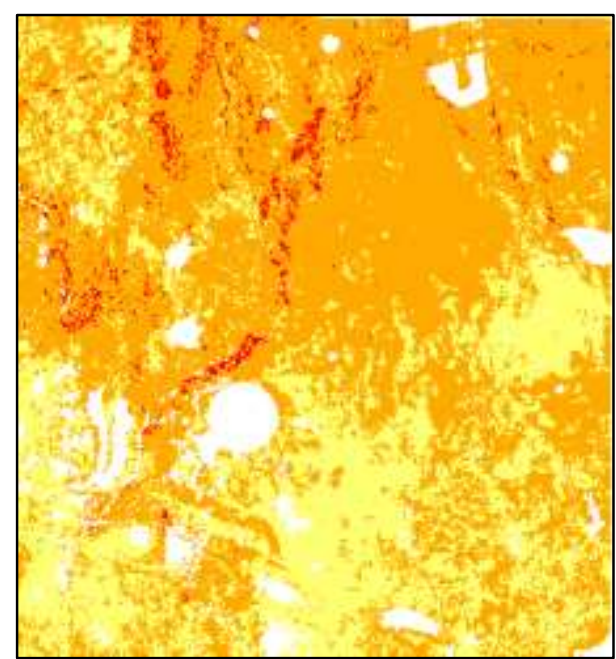

Figura 8. Susceptibilidad General calculada con momento de orden superior normalizado, $\mathrm{k}=2$.

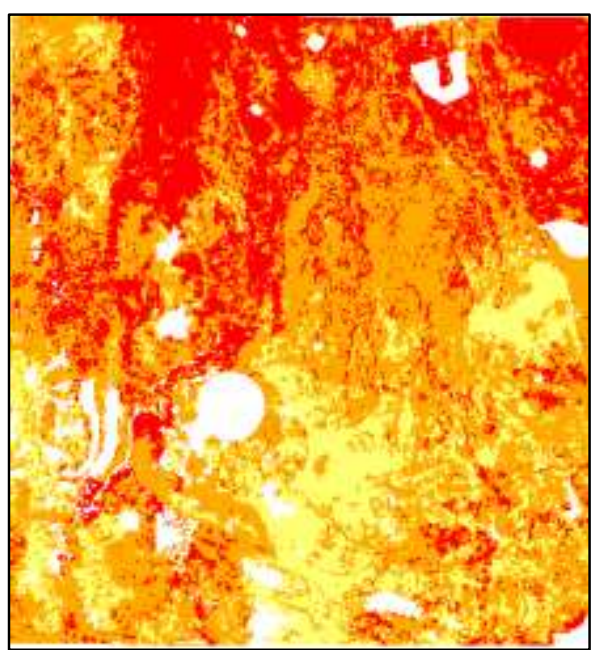

Figura 9.Susceptibilidad General calculada con momento de orden superior normalizado, k=5. 


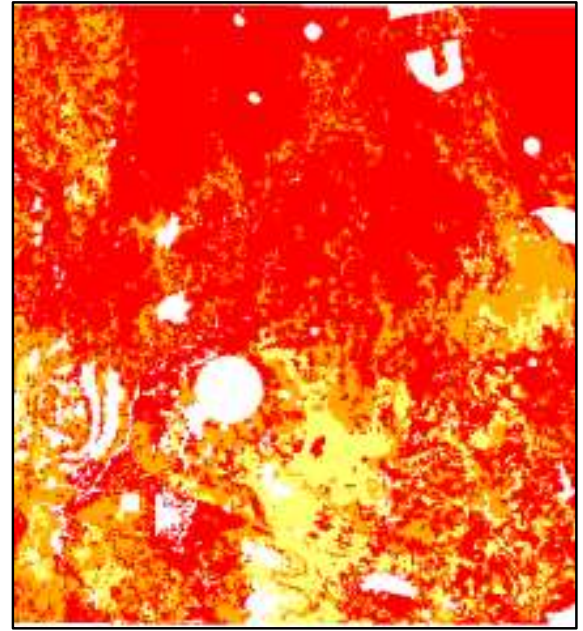

Figura 10. Susceptibilidad General calculada con momento de orden superior normalizado, k=10.

\section{Trabajos futuros}

Se sugiere, en un trabajo futuro, trabajar con momentos de orden superior centrados en 5 , considerando que los valores menores a 5 son favorables y, valores mayores a 5 , son perjudiciales. Al emplear el método con momentos de orden superior centrados se debe tener en cuenta que $\mathrm{k}$ debe ser impar, con el fin de identificar si el valor resultante de susceptibilidad es favorable o desfavorable. Este análisis proporcionaría una nueva forma de evaluar la factibilidad de un proyecto, al considerar cuando estos impactan positivamente alguna variable ambiental.

\section{Conclusiones}

De la evaluación de resultados, se concluye que el método de ponderación basado Momentos de Orden Superior Normalizado, permite visualizar mejor los diferentes efectos de cada una de las variables ambientales, aportando una superficie de susceptibilidad que permite una toma de decisiones adecuada.

El método propuesto puede ajustarse a las diferentes exigencias ambientales de la región, en la medida que se utilice un valor de potencia $\mathrm{k}$, acorde con las condiciones fisicobióticas y socioculturales de la región. La determinación del valor del coeficiente $\mathrm{k}$ depende de las condiciones del análisis, siendo mayor en la medida en que las condiciones ambientales sean mayores. Dicho de otra forma, en una región donde las condiciones ambientales no sean críticas se pueden usar valores bajos de $\mathrm{k}$ (2-5) y en zonas donde la criticidad ambiental sea alta se emplean valores de $\mathrm{k}$ altos (5-10), valores de $k$ superiores a 10, no aportan mucho pues retornan resultados muy similares al método de máximos.

El cálculo de superficies de susceptibilidad mediante funciones estadísticas que retornan el valor máximo ó el promedio aritmético o ponderado de las celdas, presentan un nivel de incertidumbre alto, debido a que no le dan la verdadera importancia a las variables que pueden tener un peso importante en la decisión o, en otros casos, no tienen en cuenta el peso de todas las variables durante el cálculo del mapa de susceptibilidad general.

La normalización de la ecuación de momentos de orden superior mediante la adición de la potencia de 1/k, permite usar valores altos de $\mathrm{k}$ sin sobrepasar el rango de análisis de los datos, lo cual facilita la interpretación de los resultados.

La superficie de susceptibilidad ambiental obtenida a partir del método de Momentos de Orden Superior Normalizado, puede ser empleado tanto en el trazado de proyectos de infraestructura lineales tales como: líneas de transmisión de energía eléctrica, carreteras, oleoductos, entre otros; al igual que en la ubicación de proyectos concentrados tales como: centrales hidroeléctricas, rellenos sanitarios, refinerías, entre otros.

De igual forma., el método puede ser empleado para calcular superficies de potencial arqueológico, minero, yacimiento de petróleo, entre otros, dependiendo de las variables analíticas empleadas.

\section{Agradecimientos}

Agradecemos la colaboración prestada por parte de la Corporación Autónoma de Antioquia CORANTIOQUIA, por la información temática facilitada para este estudio.

\section{Referencias}

[1] Ministerio de Ambiente, Vivienda y Desarrollo Territorial "Diagnóstico Ambiental de Alternativas Proyectos Lineales DA-TER3-01". Dirección de Licencias, Permisos y Trámites Ambientales, República de Colombia. Bogotá, D.C. 2006, p. 27.

[2] Ministerio de Ambiente y Desarrollo Sostenible. "Términos de Referencia para la Elaboración del Estudio de Impacto Ambiental Proyectos de Construcción y Operación de Aeropuertos Internacionales y de Nuevas Pistas". ANLA Autoridad Nacional de Licencias Ambientales. pp. 25-54, 2014

[3] Bosque Sendra, Joaquin y otro. "El uso de los Sistemas de Información Geográfica en la planificación territorial". Anales de Geografía de la Universidad Complutense. pp 49-67, 2000.

[4] Dropne Samo et al. "Multi-attribute Decision Analysis in GIS: Weighted Linear Combination and Ordered Weighted Averaging”. Informática 33, pp 459-474, 2009

[5] De Luca, Mario et al. "High-Speed Rail Track Design Using GIS And Multi-Criteria Analysis". 15th meeting of the EURO Working Group on Transportation. pp 608-617, 2012.

[6] Jacek Malczewski. "GIS-based multicriteria decision analysis: a survey of the literature". International Journal of Geographical Information Science. pp 703-726, feb 2007.

[7] Stephen J. Carver "Integrating multi-criteria evaluation with geographical information systems", International Journal of Geographical Information Systems. Pp 321-339 Abr 2007.

[8] Loro Aguayo, Manuel et al. "Optimización ambiental del trazado de corredores de carreteras con Sistemas de Información Geográfica". Libro Transporte innovador y sostenible de cara al siglo XXI. Pp 1-16. Jun 2012.

[9] Loro Aguayo, Manuel "Modelo para la Integración del trazado de infraestructuras lineales en el paisaje y el Medio Ambiente Basado en SIG". Tesis Doctoral Universidad Politécnica de Madrid. Pp 1-227, 2015.

[10] Crosetto, Michele et al. "Uncertainty and sensitivity analysis: tools for GISbased model implementation". International Journal of Geographical Information Science, pp 415-437, Agosto 2010. 
[11] Joerin, Florent et al. "Using GIS and outranking multicriteria analysis for land-use suitability assessment". International Journal of Geographical Information Science, pp 153-174, Agosto 2010.

[12] Jacomina Heymans, Johanna et al. "Best practice in Ecopath with Ecosim food-web models forecosystem-based management". Ecological Modelling. Pp 173-184, Enero 2016.

[13] Kim de Mutsert et al. "Exploring effects of hypoxia on fish and fisheries in the northern Gulfof Mexico using a dynamic spatially explicit ecosystem model". Ecological Modelling. pp 142-150, Noviembre 2015.

[14] Russi Navarrete, Gustavo Adolfo. Gestión ambiental para la ejecución de obras civiles. Revista Ingenierías Universidad de Medellín. No. 2. pp 112-116, Junio 2003.

[15] L. Dini, "Apuntes de probabilidad y estadística" pp 12-14. 2003. (Disponible http://www.csanpablo.com.ar/apuntes_archivos/fisica_archivos/probab ilidad_y_\%20estadistica.PDF ).

[16] Rodríguez Becerra, Manuel; Uribe Botero, Eduardo; Carrisoza Umaña, Instrumentos económicos para la gestión ambiental en Colombia. Santafé de Bogotá: Grupo Editorial 87, 146 p. (CEREC: Serie Ecológica No 10), 1996.

[17] SANINT, Enrique Angel. Métodos cuantitativos para la toma de decisiones ambientales. 2 Ed. Medellín : Universidad Nacional de Colombia, Pp 156. 2000

[18] EVANS, MICHAEL et al. Probabilidad y Estadística. 2 Ed. Reverte S.A. Barcelona España, Pp 184-186. 2004

[19] Pernas Sanches, Isaac. Matemáticas de las Operaciones Financieras. Ed Isaac Pernas. Madrid España, pp. 115-117. 2011

[20] Universidad de la Habana. Revista de la Facultad de Ciencias y Letras. Vol 3, Pp 57. 1906 